\title{
Effects of Eupatilin on Insulin-Like Growth Factor 1-Induced Lipogenesis and Inflammation of SZ95 Sebocytes
}

\author{
Ji Hyun Lee, Ye Jin Lee, Ji Young Song ${ }^{1}$, Yeong Ho Kim, Jun Young Lee, Christos C. Zouboulis ${ }^{2}$, \\ Young Min Park \\ Department of Dermatology, Seoul St. Mary's Hospital, College of Medicine, The Catholic University of Korea, ${ }^{1}$ Department of \\ Biomedicine \& Health Sciences, College of Medicine, The Catholic University of Korea, Seoul, Korea, ${ }^{2}$ Departments of Dermatology, \\ Venereology, Allergology and Immunology, Dessau Medical Center, Dessau, Germany
}

\section{Dear Editor:}

Acne is inflammatory disorder of the pilosebaceous unit. Its mechanism is complex, occurring as a result of hyperkeratinization of the pilosebaceous ducts, colonization of Propionibacterium acnes, and perifollicular inflammation with an imbalance of sebum production ${ }^{1}$. Insulin-like growth factor (IGF)-1 and peroxisome proliferator-activated receptor (PPAR) regulate the growth and differentiation of sebocytes and sebum secretion. Hyperglycemic diets induce IGF-1 production and may aggravate acne ${ }^{2}$. Eupatilin [2-(3,4-dimethoxyphenyl)-5,7-dihydroxy-6-methoxychromen-4-one] is the main flavonoid of the Artemisia species. Eupatilin exerts various effects including anti-apoptotic, cytoprotective, antioxidant, and anti-inflammatory nature on many different cell lines ${ }^{3}$. Eupatilin demonstrates antioxidant activity by suppressing reactive oxygen species and anti-inflammatory activity by inhibiting 5-lipoxygenase ${ }^{4}$. In addition, eupatilin downregulates the production of inflammatory cytokines such as tumor necrosis factor (TNF)- $\alpha$, interleukin (IL)-4, IL-6, and IL-18 through nuclear factor kappa B (NF- $\kappa$ B) in RBL-2H3 cells ${ }^{3-5}$. Eupatilin has been used in an attempt to address inflammatory diseases, but its therapeutic effect on acne and related mechanisms remain unclear. In this study, we aimed to investigate the therapeutic effect of eupatilin on IGF-1-induced in- flammation and lipogenesis using human sebocytes.

To examine the effects of eupatilin in vitro, we used human SZ95 sebocytes (Supplementary Material). The effects of eupatilin on the proliferation of cultured SZ95 sebocytes were determined with various doses of eupatilin. In MTT assay, the proliferation of SZ95 sebocytes was sequentially decreased according to the concentration (10, 20, 50, or $100 \mu \mathrm{M}$ of eupatilin) (Supplementary Material). Thus, we determined experimental concentration as 10- and $100-\mu \mathrm{M}$ of eupatilin. Next, we stained an intracellular lipid droplet formation using Oil Red O to investigate the effects of eupatilin on the lipid synthesis of sebocytes. When SZ95 sebocytes were treated with eupatilin, lipid accumulation in the cytoplasm was significantly reduced (Fig. 1A, B). To elucidate how eupatilin suppressed IGF-1-induced lipogenesis of SZ95 sebocytes, the effects of eupatilin treatment on the IGF-1-induced expression levels of phosphorylated Akt and lipogenesis-related transcription factors (PPAR $\gamma$ and mature sterol regulatory element-binding protein [SREBP]-1) were measured. In comparison with no treatment, $100 \mu \mathrm{g} / \mathrm{ml}$ of eupatilin significantly reduced the protein levels of phosphorylated Akt, PPAR $\gamma$, and mature SREBP-1 of the sebocytes, which were increased by IGF-1 pretreatment (Fig. 1C). Likewise, eupatilin also significantly downregulated the mRNA ex-

\footnotetext{
Received August 28, 2018, Revised October 18, 2018, Accepted for publication December 12, 2018
}

Corresponding author: Young Min Park, Department of Dermatology, Seoul St. Mary's Hospital, College of Medicine, The Catholic University of Korea, 222 Banpo-daero, Seocho-gu, Seoul 06591, Korea. Tel: 82-2-2258- 6223, Fax: 82-2-594-3255, E-mail: yymmpark6301@hotmail.com ORCID: https://orcid.org/0000-0002-3631-0807

This is an Open Access article distributed under the terms of the Creative Commons Attribution Non-Commercial License (http://creativecommons.org/ licenses/by-nc/4.0) which permits unrestricted non-commercial use, distribution, and reproduction in any medium, provided the original work is properly cited.

Copyright (c) The Korean Dermatological Association and The Korean Society for Investigative Dermatology 
A

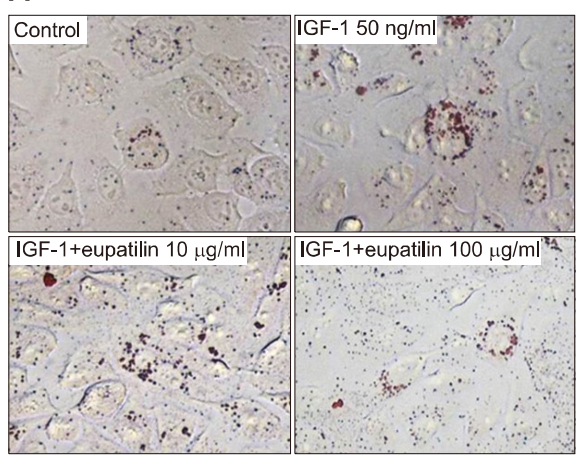

D

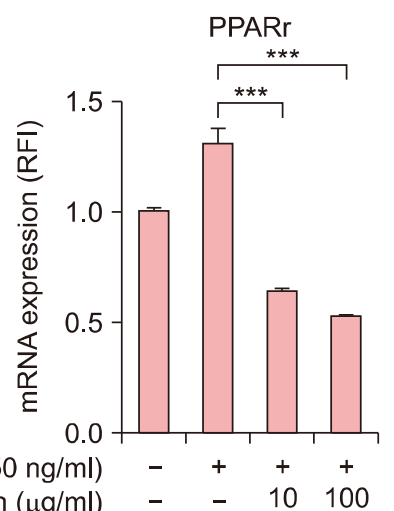

B

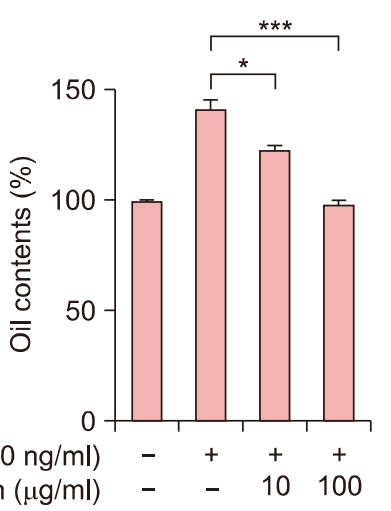

SREBP-1a

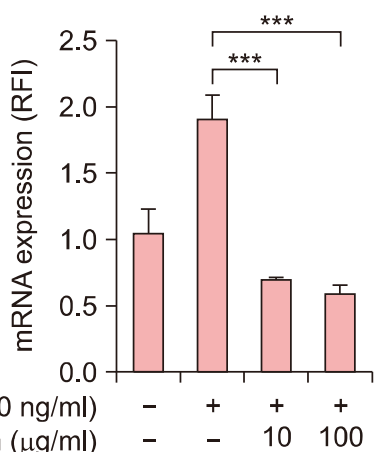

C
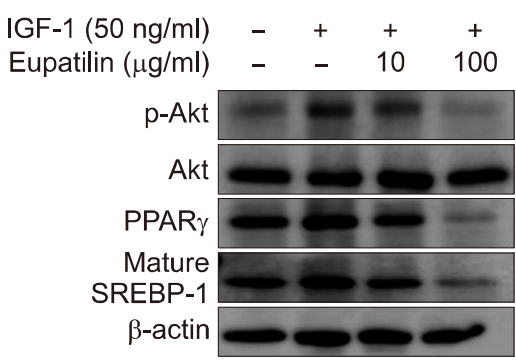

SREBP-1c

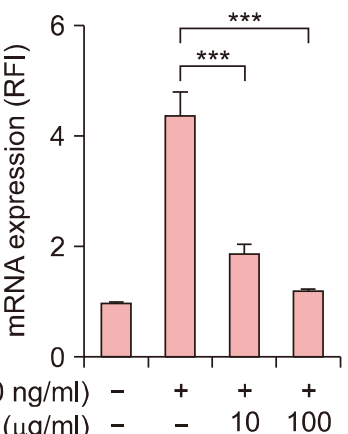

Fig. 1. Effects of eupatilin on the intracellular lipid synthesis of SZ95 sebocytes. With the exception of the control group, SZ95 sebocytes were pretreated with $50 \mathrm{ng} / \mathrm{ml}$ of insulin-like growth factor (IGF)-1 for 48 hours and then with $10 \mu \mathrm{g} / \mathrm{ml} \mathrm{or} 100 \mu \mathrm{g} / \mathrm{ml}$ of eupatilin for 48 hours. (A) Intracellular lipid droplets of SZ95 sebocytes treated with eupatilin were detected by Oil Red O staining. Bars $=20$ $\mu \mathrm{m}$. (B) Supernatant Oil Red O levels (\%) were measured by their optical density at $500 \mathrm{~nm}$. (C) Whole-cell lysates were prepared and analyzed by western blotting. Blots were incubated with antibodies specific for total and phosphorylated forms of Akt, peroxisome proliferator-activated receptor (PPAR)- $\gamma$, and mature sterol regulatory element-binding protein (SREBP)-1. (D) Quantitative reversetranscription polymerase chain reaction of PPAR $\gamma$, SREBP-1a, and SREBP-1c for the evaluation of mRNA expression was performed. Data are presented as the mean \pm standard error of triplicate assay $(\mathrm{n}=5)$. Data were analyzed using the Student's t-test $(* p<0.05$, $* * * p<0.001)$. RFI: relative fold increase.

pression level of PPAR $\gamma$, SREBP-1a, and SREBP-1c of the sebocytes (Fig. 1D). These results suggest that eupatilin has an inhibitory effect on IGF-I-induced lipogenesis of sebocytes through suppression of the phosphorylation of Akt, PPAR $\gamma$, and mature SREBP-1.

To further investigate the anti-inflammatory effects of eupatilin on sebocytes, we analyzed the IGF-1-induced proinflammatory cytokines of sebocytes. In the immunofluorescence study, treatment with eupatilin inhibited the translocation of NF- $\kappa$ B p65 induced by $50 \mathrm{ng} / \mathrm{ml}$ of IGF-1 (Fig. 2A). This eupatilin treatment also significantly downregulated the mRNA expression levels of (pro)inflammatory cytokines such as TNF- $\alpha$, IL-6, and IL-8 (Fig. 2B). In this study, we first showed that the proper concentrations of eupatilin $(10 \mu \mathrm{g} / \mathrm{ml}$ and $100 \mu \mathrm{g} / \mathrm{ml})$ had potent anti-IGF-I effects on SZ95 sebocytes, as follows: 1) eupatilin suppressed the lipogenesis of sebocytes by interfering with the expression of phosphorylated Akt, $\operatorname{PPAR} \gamma$, and SREBP-1 induced by IGF-1; and 2) eupatilin reduced the inflammatory response of sebocytes by inhibiting NF- $\kappa$ B activation, with IGF-1 able to induce the inflammatory cytokine expression of sebocytes.

SREBP-1 is a major transcription factor that regulates cholesterol/fatty acid metabolism ${ }^{6}$. Smith et al. ${ }^{7}$ reported that IGF-1 induced SREBP-1. This activation occurred via a phosphoinositide 3-kinase (PI3K)/Akt pathway. They also showed that IGF-I transmits its lipogenic signal in sebocytes via an Akt pathway. Meanwhile, PPAR $\gamma$ is a potential modulator of lipid production in human sebocytes ${ }^{8}$. PPARs are regulators of lipogenesis and differentiation of keratinocytes as well as sebocytes and have three isoforms (i.e., $\alpha, \delta$, and $\gamma$ ). Notably, PPAR $\gamma$ is important for sebaceous gland development and function ${ }^{9}$. Interestingly, in the present study, the Oil Red O stain showed that eupatilin suppressed lipid synthesis by sebocytes. Although eupatilin from Artemisia plants has been reported as a se- 
A

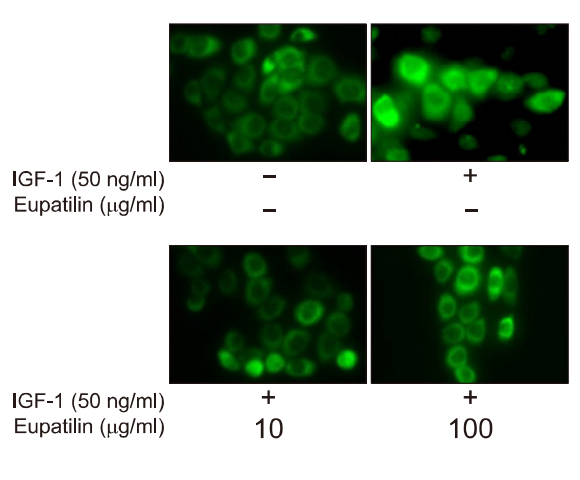

B

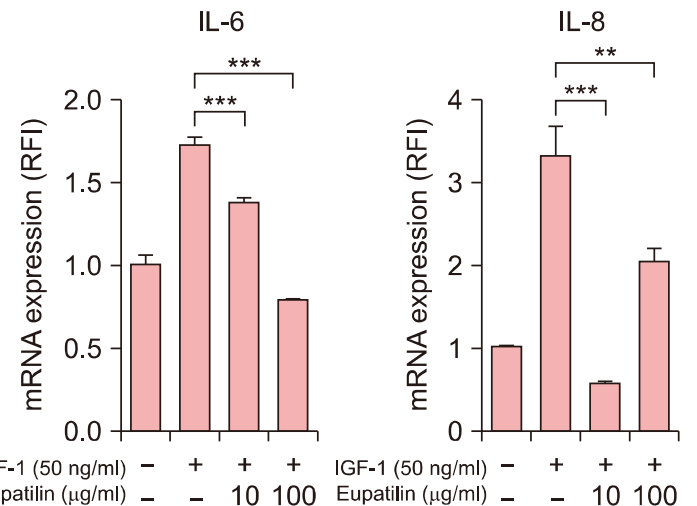

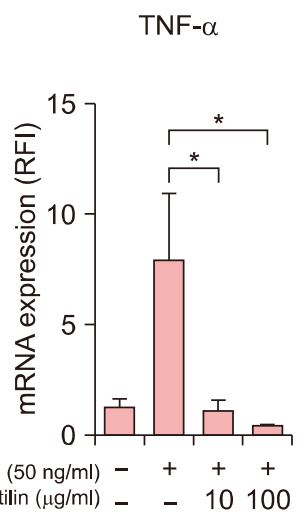

Fig. 2. Inhibition of insulin-like growth factor (IGF)-1-induced (pro)inflammatory cytokines of SZ95 sebocytes by eupatilin. With the exception of the control group, SZ95 sebocytes were treated with $50 \mathrm{ng} / \mathrm{ml}$ of IGF-1 for 48 hours and then with $10 \mu \mathrm{g} / \mathrm{ml}$ and $100 \mu \mathrm{g} / \mathrm{ml}$ of eupatilin for 48 hours. (A) Cells were incubated with primary antibody (nuclear factor kappa B [NF- $\kappa$ B] p65) and were finally visualized under a fluorescence microscope. (B) Quantitative Reverse-transcription polymerase chain reaction for the evaluation of mRNA expression of tumor necrosis factor (TNF)- $\alpha$, interleukin (IL)-6, and IL-8 was performed. Data are presented as the mean \pm standard error of triplicate assay $(\mathrm{n}=5)$. Data were analyzed using the Student's t-test $\left({ }^{*} p<0.05,{ }^{* *} p<0.01, * * * p<0.001\right)$. RFI: Relative fold increase.

lective PPAR $\alpha$ agonist $^{10}$, in the present study, the proper concentrations of eupatilin $(10 \mu \mathrm{g} / \mathrm{ml}$ and $100 \mu \mathrm{g} / \mathrm{ml})$ inhibited the IGF-1-induced SREBP-1 and PPAR $\gamma$ expression of sebocytes. In addition, eupatilin inhibited the phosphorylation of Akt. This inhibitory effect of eupatilin on IGF-1-induced sebocytes was confirmed by the reduced mRNA expression level of PPAR $\gamma$, SREBP-1a, and SREPB-1c. Moreover, we found that eupatiln suppressed the mRNA expression of TNF- $\alpha$, IL-6, and IL-8 of sebocytes at the transcription level. Likewise, eupatilin also inhibited IGF1 -induced nuclear translocation of NF- $\kappa$ B p65 of sebocytes. This study demonstrate that eupatilin strongly downregulated IGF-I-induced inflammation and lipogenesis of SZ95 sebocytes. Therefore, our results suggest that eupatilin could be a promising candidate for the management of acne.

\section{ACKNOWLEDGMENT}

This work was supported by a National Research Foundation of Korea (NRF) grant funded by the Korean government (MSIP) (no. NRF-2015R1C1A2A01054767 and 2018 R1D1A1B07044100) and by Research Fund of Seoul St. Mary's Hospital, The Catholic University of Korea. And This research was supported by a grant from the Korea Health Technology R\&D Project through the Korea Health Industry Development Institute (KHIDI) funded by the Ministry of Health \& Welfare of the Republic of Korea (no. HI14C2116).

\section{SUPPLEMENTARY MATERIALS}

Supplementary data can be found via http://anndermatol. org/src/sm/ad-31-479-s001.pdf.

\section{CONFLICTS OF INTEREST}

The authors have nothing to disclose.

\section{ORCID}

Ji Hyun Lee, https://orcid.org/0000-0002-3671-502X

Ye Jin Lee, https://orcid.org/0000-0002-9600-8220

Ji Young Song, https://orcid.org/0000-0002-5749-3032

Yeong Ho Kim, https://orcid.org/0000-0003-4983-4906

Jun Young Lee, https://orcid.org/0000-0002-8650-1759

Christos C. Zouboulis, https://orcid.org/0000-0003-1646-2608

Young Min Park, https://orcid.org/0000-0002-3631-0807

\section{REFERENCES}

1. Zouboulis CC. Acne and sebaceous gland function. Clin Dermatol 2004;22:360-366.

2. Melnik BC, Schmitz G. Role of insulin, insulin-like growth factor-1, hyperglycaemic food and milk consumption in the pathogenesis of acne vulgaris. Exp Dermatol 2009;18:833841.

3. Lee JH, Lee YJ, Lee JY, Park YM. Topical application of Eupatilin ameliorates atopic dermatitis-like skin lesions in NC/Nga mice. Ann Dermatol 2017;29:61-68.

4. Choi EJ, Oh HM, Na BR, Ramesh TP, Lee HJ, Choi CS, et al. 
Eupatilin protects gastric epithelial cells from oxidative damage and down-regulates genes responsible for the cellular oxidative stress. Pharm Res 2008;25:1355-1364.

5. Lee SH, Bae EA, Park EK, Shin YW, Baek NI, Han EJ, et al. Inhibitory effect of Eupatilin and jaceosidin isolated from Artemisia princeps in IgE-induced hypersensitivity. Int Immunopharmacol 2007;7:1678-1684.

6. Engelking LJ, Kuriyama $\mathrm{H}$, Hammer RE, Horton JD, Brown MS, Goldstein JL, et al. Overexpression of Insig-1 in the livers of transgenic mice inhibits SREBP processing and reduces insulin-stimulated lipogenesis. J Clin Invest 2004; 113:1168-1175.

7. Smith TM, Gilliland K, Clawson GA, Thiboutot D. IGF-1 induces SREBP-1 expression and lipogenesis in SEB-1 sebo- cytes via activation of the phosphoinositide 3-kinase/Akt pathway. J Invest Dermatol 2008;128:1286-1293.

8. Trivedi NR, Cong Z, Nelson AM, Albert AJ, Rosamilia LL, Sivarajah $S$, et al. Peroxisome proliferator-activated receptors increase human sebum production. J Invest Dermatol 2006; 126:2002-2009.

9. Ferré P. The biology of peroxisome proliferator-activated receptors: relationship with lipid metabolism and insulin sensitivity. Diabetes 2004;53 Suppl 1:S43-S50.

10. Choi $Y$, Jung $Y$, Kim SN. Identification of Eupatilin from Artemisia argyi as a selective PPARa agonist using affinity selection ultrafiltration LC-MS. Molecules 2015;20:1375313763.

\title{
Can Body Mass Index and/or Waist Circumference Be the Risk Factors of Chronic Spontaneous Urticaria?: A Nationwide Population-Based Study
}

\author{
Yoon Seob Kim, Kyungdo Han ${ }^{1}$, Ji Hyun Lee, Jun Young Lee, Young Min Park \\ Department of Dermatology, Seoul St. Mary's Hospital, The Catholic University of Korea, ${ }^{1}$ Department of Medical Statistics, College of \\ Medicine, The Catholic University of Korea, Seoul, Korea
}

Dear Editor:

Several studies have suggested an association between chronic spontaneous urticaria (CSU) and body mass index $(\mathrm{BMI})^{1-3}$ or metabolic syndrome ${ }^{1,4}$. In contrast, a French study reported that obesity was not associated with severe $\mathrm{CSU}^{5}$. There is little evidence that waist circumference (WC), another scale which correlates well with visceral obesity, is associated with CSU. We hypothesized that obesity could be associated with increased CSU risk. The aim of our study was to investigate the impact of BMI and/or WC on the risk for CSU in an adult Korean population using a nationwide database. The study was approved by the Institutional Review Board of The Catholic University of Korea (IRB no. KC16EISE0852).

The health check-up database, a sub-dataset of the Korean National Health Insurance Service (NHIS) database (2002 2015), was used for data collection. NHIS subscribers are advised to have biannual health check-ups including height,

Received September 13, 2018, Revised November 15, 2018, Accepted for publication January 2, 2019

Corresponding author: Young Min Park, Department of Dermatology, Seoul St. Mary's Hospital, College of Medicine, The Catholic University of Korea, 222 Banpo-daero, Seocho-gu, Seoul 06591, Korea. Tel: 82-2-2258-6223, Fax: 82-2-599-9950, E-mail: yymmpark6301@hotmail.com ORCID: https://orcid.org/0000-0002-3631-0807

This is an Open Access article distributed under the terms of the Creative Commons Attribution Non-Commercial License (http://creativecommons.org/ licenses/by-nc/4.0) which permits unrestricted non-commercial use, distribution, and reproduction in any medium, provided the original work is properly cited.

Copyright $\odot$ The Korean Dermatological Association and The Korean Society for Investigative Dermatology 


\section{Supplementary Materials}

\section{MATERIALS AND METHODS}

\section{Cells and reagents}

Immortalized human SZ95 sebocytes (provided by Prof. Christos C. Zouboulis) were maintained in Sebomed ${ }^{\circledR}$ basal medium (Biochrom $\mathrm{GmbH}$, Berlin, Germany) containing 10\% (v/v) fetal bovine serum (Gibco; Invitrogen, Carlsbad, CA, USA), $5 \mathrm{ng} / \mathrm{ml}$ of human recombinant epidermal growth factor (Invitrogen, Grand Island, NY, USA), 50 IU/ml of penicillin, and $50 \mu \mathrm{g} / \mathrm{ml}$ of streptomycin (Gibco) in a humidified atmosphere containing $5 \% \mathrm{CO}_{2}$ at $37^{\circ} \mathrm{C}$ (Zouboulis et al., 1999) ${ }^{1}$. Culture medium was replaced every two days. Eupatilin was provided by Dong-A Pharmaceutical Co., Ltd. (Yongin, Korea) and dissolved in 10\% dimethyl sulfoxide. The experimental design is shown in Supplementary Fig. 1.

\section{Determination of cell proliferation by MTT assay}

Cell proliferation was analyzed by by 3-(4,5-dimethylthiazol-2-yl)-2,5-diphenyl tetrazolium bromide MTT assay. SZ95 human sebocytes were seeded $\left(1 \times 10^{3}\right.$ cells per well) in triplicate into 96-well plates, incubated overnight, and then treated with eupatilin at the concentrations of $10 \mu \mathrm{g} / \mathrm{ml}, 20 \mu \mathrm{g} / \mathrm{ml}, 50 \mu \mathrm{g} / \mathrm{ml}$, and $100 \mu \mathrm{g} / \mathrm{ml}$ (Supplementary Fig. 2) in Sebomed $^{\mathbb{R}}$ media (Biochrom $\mathrm{GmbH}$ ) without serum at $37^{\circ} \mathrm{C}$ in $5 \% \mathrm{CO}_{2}$. Subsequently, $100 \mu \mathrm{l}$ of MTT at $5 \mathrm{mg} / \mathrm{ml}$ was added to each well and incubation was continued for 4 hours. Supernatants were removed and formazan crystals resulting from mitochondrial enzymatic activity on the MTT substrate were solubilized with dimethyl sulfoxide (Sigma-Aldrich, St. Louis, MO, USA). The absorbance was measured at $540 \mathrm{~nm}$ using an enzyme-linked immunosorbent assay reader (VersaMax; Molecular Devices, Sunnyvale, CA, USA).

\section{Oil Red $O$ staining and lipid detection}

SZ95 sebocytes were seeded $\left(1 \times 10^{4}\right.$ cells $\left./ \mathrm{ml}\right)$ on 12-well culture plates, incubated overnight, and then treated with $50 \mu \mathrm{g} /$ $\mathrm{ml}$ of IGF-1 and $10 \mu \mathrm{g} / \mathrm{ml}$ or $100 \mu \mathrm{g} / \mathrm{ml}$ of eupatilin for 48 hours. Cells treated with the vehicle served as controls. At the end of the treatment period, cells were washed with phosphate-buffered saline and fixed through incubation in $10 \%$ formalin for one hour at room temperature. Fixed cells were incubated with $60 \%$ isopropanol for five minutes and then isopropanol was completely removed by air-dry. Cells were stained for 10 minutes with filtered Oil Red O working solution, prepared immediately before use by making a 6:4 mixture of stock $(0.5 \%$ Oil Red O from Sigma-Aldrich in $99 \%$ isopropanol) and $\mathrm{dH}_{2} \mathrm{O}$. Supernatant Oil Red O levels were measured by measuring the optical density at $500 \mathrm{~nm}$.

\section{Western blot analysis}

Cells were lysed in lysis buffer containing a protease and phosphatase inhibitor cocktail (Thermo Fisher Scientific, Waltham, MA, USA). Equal amounts (30 $\mu \mathrm{g}$ ) of extracted protein were resolved using $6 \%$ to $12 \%$ sodium dodecyl sulfatepolyacrylamide gel electrophoresis and transferred to polyvinylidene difluoride membranes. Following incubation in blocking solution, the membranes were incubated overnight at $4{ }^{\circ} \mathrm{C}$ with the appropriate antibodies. Blots were then incubated with peroxidase-conjugated secondary antibodies and visualized by enhanced chemiluminescence substrate (Thermo Fisher Scientific). The following primary antibodies were used: phospho-Akt, Akt, peroxisome proliferator-activated receptor (PPAR) $\gamma$, mature sterol regulatory element-binding protein (SREBP)-1 (Cell Signaling Technology, Danvers, MA, USA), and actin (Santa Cruz Biotechnologies, Dallas, TX, USA).

\section{Immunofluorescence}

SZ95 sebocytes were grown on cell culture slides (SPL Life Sciences, Pocheon, Korea), fixed through incubation with $4 \%$ paraformaldehyde for 20 minutes, and permeabilized through incubation with $0.1 \%$ Triton X-100 in phosphate-buffered saline for 10 minutes at room temperature. Cells were then incubated overnight at $4{ }^{\circ} \mathrm{C}$ with primary antibody (i.e., nuclear factor kappa B [NF- $\kappa$ B] p65) (Santa Cruz Biotechnologies) and then for one hour at room temperature with fluorescein isothiocyanate-conjugated secondary antibody (Santa Cruz Biotechnologies), and were finally visualized under a fluorescence 
Brief Report

microscope (Olympus, Tokyo, Japan).

\section{Reverse-transcription polymerase chain reaction}

To evaluate gene expression, total RNA was isolated using Trizol ${ }^{\mathbb{R}}$ Reagent (Invitrogen) according to the manufacturer's protocols. Equal amounts of RNA $(1 \mu \mathrm{g})$ were reverse-transcribed into complementary DNA using the Prime Script ${ }^{\mathrm{TM}}{ }_{\mathrm{RT}}$ reagent Kit with gDNA Eraser ${ }^{\circledR}$ (Takara Bio, Otsu, Japan). Quantitative reverse-transcription polymerase chain reaction (RT-PCR) was performed using a CFX96 ${ }^{\text {TM }}$ Real-Time PCR Detection System ${ }^{\circledR}$ (Bio-Rad, Hercules, CA, USA) with SYBR ${ }^{\mathbb{R}}$ premix EX Taq ${ }^{\text {TM }}$ (Takara Bio, Kusatsu, Japan) and specific primers for tumor necrosis factor (TNF)- $\alpha$, interleukin (IL)-6, IL-8, PPAR- $\gamma$, SREBP-1a, and SREBP-1c. The cycling conditions consisted of an initialization step for 10 seconds at $95^{\circ} \mathrm{C}$ followed by two-step PCR for 40 cycles of $95^{\circ} \mathrm{C}$ for five seconds (denaturation) and $58^{\circ} \mathrm{C}$ to $60^{\circ} \mathrm{C}$ for 30 seconds (annealing/extension). Fluorescence intensity was measured in real time using the optical module. Melt curves were used to determine product specificity. Results were normalized to the level of glyceraldehyde 3-phosphate dehydrogenase gene expression. The analysis of relative gene expression data was conducted using the $2^{-\Delta \Delta \mathrm{CT}}$ method. All experiments were repeated twice. The primers used are as follows : TNF- $\alpha$, forward 5'-CCCAGGGACCTCTCTCTAATC-3' and reverse 5'ATGGGCTACAGGCTTGTCACT-3'; IL-6, forward 5'-ACCCCCAATAAATATAGGACTGGA-3' and reverse 5'-GAGAAGGC AACTGGACCGAA-3'; IL-8, forward 5'-GGTGCAGTTTTGCCAAGGAG-3' and reverse 5'-TGGGGTGGAAAGGTTTGGAG$3^{\prime}$; PPAR- $\gamma$, forward 5'-GCCCAGGTTTGCTGAATGTG-3' and reverse 5'-TGAGGACTCAGGGTGGTTCA-3'; and SREBP1a, forward 5'-GCTGCTGACCGACATCGAA-3', SREBP-1c, forward 5'-GGAGCCATGGATTGCACTTT-3', and SREBP-1a,c, reverse 5'-TCAAATAGGCCAGGGAAGTCA-3'.

\section{Statistical analysis}

All data were expressed as the mean \pm standard error of the mean. One-way analysis of variance followed by Tukey's multiple comparison test was used for statistical analysis. The Kruskal-Wallis test was used for comparisons of the four groups. Statistical significance was set at $p<0.05$.

\section{REFERENCE}

1. Zouboulis CC, Seltmann H, Neitzel H, Orfanos CE. Establishment and characterization of an immortalized human sebaceous gland cell line (SZ95). J Invest Dermatol 1999;113:1011-1020. 
Brief Report

Growth medium

Supplementary Fig. 1. Experimental design. IGF-1: insulin-like growth factor-1. 
Brief Report

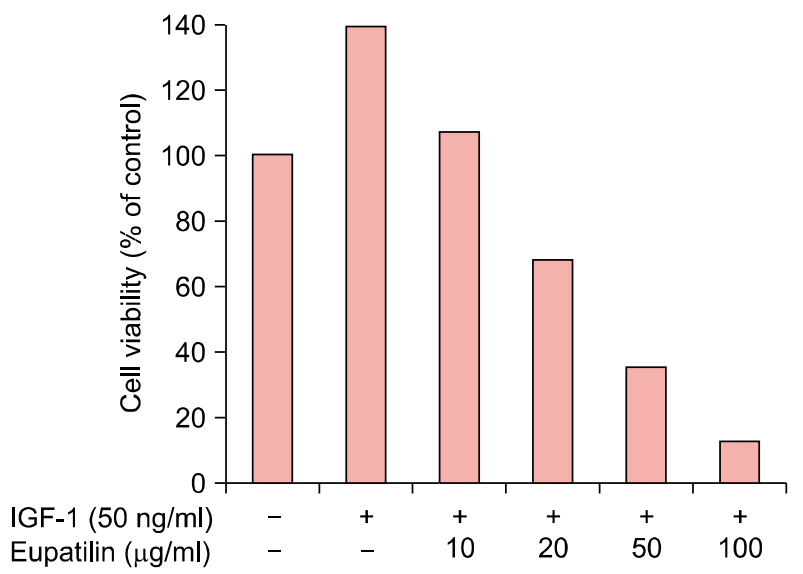

Supplementary Fig. 2. The effects of eupatilin on cell proliferation by MTT assay. The effect of eupatilin on SZ95 sebocyte proliferation was determined by MTT assay. Cells were treated with various concentrations of eupatilin $(10 \sim 100 \mu \mathrm{M})$. IGF-1: insulin-like growth factor-1. 